\title{
A SHORT PROOF OF CURRY'S NORMAL FORM THEOREM
}

\section{ROGER HINDLEY AND BRUCE LERCHER ${ }^{1}$}

In Chapter 6 of their book [1] Curry and Feys define a notion of reduction (strong reduction) for the extensional theory of equality in combinatory logic, show [1, Theorem 3, p. 221] that strong reduction has the Church-Rosser property, and define a notion of normal form in analogy with the corresponding concept in lambda-conversion. Curry's normal form theorem [1, Theorem 7, p. 230] asserts that if a term ("ob") of combinatory logic is in normal form, it is irreducible, so that if $X$ has normal form $X^{*}$, then $X$ reduces to $X^{*}$ by a process (namely, strong reduction) that cannot be continued further.

Curry's proof of his theorem in [1] is quite long and difficult (see $[3$, p. 228] for comment). There is another lengthy proof in the current draft of [2] and the first author has discovered a proof using his axiomatization of strong reduction [3]. The present proof follows the same general line as the latter proof, but it is considerably shorter and simpler.

Definitions and notation are as in [3], except for the symbol $\triangleright$ which is used here for weak reduction.

1. Substitution and abstraction. The first result is essentially from [4, Lemmas 1 and 4]. The proofs, by induction, are easy.

Lemma 1. Let $P$ be a redex scheme. Then:

(a) $P$ contains at most one occurrence of each meta-variable;

(b) If $M$ is a meta-variable occurring in $P$, there is an $N$ such that $N M$ occurs in $P$;

(c) If $P$ is not basic (i.e. is the result of at least one application of scheme (viii) of $[3$, p. 233]), then $P$ is weakly irreducible;

(d) If $P$ is not basic, then either $P \equiv \mathbf{S} P_{1} P_{2}$ or $P \equiv \boldsymbol{S} P_{1}$ where $P_{1}$ contains at least one occurrence of an atomic combinator.

The hypotheses of the next lemma are, in essence, the properties of redex schemes asserted in Lemma 1(a), (b), and (c).

Received by the editors December 21, 1968.

1 The second author was supported in part by The Research Foundation of State University of New York, under fellowship FRF 68-40-031. This paper was written while he was on sabbatical leave at University College of Swansea, Wales, whose Department of Pure Mathematics he wishes to thank for their hospitality. 
Lemma 2. Suppose that $P$ is weakly irreducible, that $P$ contains at most one occurrence of each meta-variable, and that either $P$ is itself a meta-variable, or the occurrence of any meta-variable $M$ in $P$ is in a component $N M$ of $P$. If $[X / A][Y / B][Z / C] P \triangleright R$, then $R \equiv\left[X^{\circ} / A\right]$ - $\left[Y^{\circ} / B\right]\left[Z^{\circ} / C\right] P$, where $X \triangleright X^{\circ}, Y \triangleright Y^{\circ}$, and $Z \triangleright Z^{\circ}$.

Proof. The proof is by induction on $P$, with three basic clauses: $P \equiv M ; P \equiv N M$, where $N$ does not contain any meta-variables; and $P$ does not contain any meta-variables. The induction step, $P \equiv P_{1} P_{2}$, uses the fact that no substitution instance of $P$ is itself a weak redex.

The next result is Lemma 11 of [3].

Lemma 3. Let $P$ be a redex scheme and suppose that $U, V, W$ do not contain $x$. Then $[U / A][V / B][W / C][x] P_{i j k} \equiv[x][U / A][V / B]$ . $[W / C] P_{i j k} \equiv[x]\left[U^{i} / A\right]\left[V^{j} / B\right]\left[W^{k} / C\right] P$.

The final preliminary result is an immediate consequence of the Church-Rosser Theorem for weak reduction (see, e.g. [2], or [5, Theorem 12]). It can also be proved directly by induction on $Y$.

Lemma 4. If $Y$ is weakly irreducible, $X \equiv[x] Y$, and $X x \triangleright Q$, then $Q \triangleright Y$.

2. The normal form theorem. By [3], $X$ is irreducible if it contains no redexes, so Curry's theorem may be stated as follows.

NORMAL FORM THEOREM. If $X$ is in normal form, it contains no redexes.

Proof. The proof is by induction on the definition of normal form.

(1) If $X \equiv x$, then $X$ contains no redex by Lemma 1(b) or (d).

(2) If $X \equiv x X_{1} \cdots X_{n}$, with $X_{1}, \cdots, X_{n}$ in normal form, assume by induction that $X_{1}, \cdots, X_{n}$ contain no redexes. The only possible redexes in $X$ then have $x$ at the head, which is impossible by Lemma $1(d)$.

(3) If $X \equiv[x] Y$, where $Y$ is in normal form, assume by induction that $Y$ contains no redexes. The rest of the proof is by induction on $Y$. Note that $Y$ is weakly irreducible.

(3a) If $Y \equiv x$, then $X \equiv I$, which is not a redex by Lemma 1(d).

(3b) If $Y$ does not contain $x$, then $X \equiv K Y$. By Lemma 1(d), neither $X$ nor $\boldsymbol{K}$ is a redex, so any redexes in $X$ must be components of $Y$.

(3c) If $Y \equiv X x$ and contains no redexes, clearly $X$ contains none.

(3d) If $Y \equiv Y_{1} Y_{2}$ and $X \equiv \mathbf{S} X_{1} X_{2}$ where $X_{i} \equiv[x] Y_{i}, i=1,2$, then assume the theorem for $X_{1}$ and $X_{2}$. Then the only possible redexes in 
$X$ (by Lemma $1(\mathrm{~d})$ ) are $\boldsymbol{S} X_{1}$ and $X$ itself. Consider the two cases separately.

(3d1) Suppose $\boldsymbol{S} X_{1}$ is a redex. Then $\boldsymbol{S} X_{1}$ is a substitution instance of a redex scheme $\boldsymbol{S} R$. If $\boldsymbol{S} R$ is basic, then $\boldsymbol{S} R \equiv \boldsymbol{S}(\boldsymbol{K I})$, so $\boldsymbol{K I} \equiv[x] Y_{1}$. Hence $Y_{1} \equiv I$, contradicting the hypothesis that $Y$ is weakly irreducible. Thus, $\boldsymbol{S} R \equiv[x] P_{i j k}$ for a redex scheme $P$. This implies that $P \equiv \boldsymbol{S} R M$ for a meta-variable $M$, so that $X$ itself is also a redex. Hence, this case may be included in the next.

(3d2) Suppose $X \equiv \boldsymbol{S} X_{1} X_{2}$ is a redex, a substitution instance of a redex scheme $\mathbf{S} R_{1} R_{2}$. If $\mathbf{S} R_{1} R_{2}$ is basic, then $R_{1} \equiv \boldsymbol{K} A$, so $X_{1} \equiv \boldsymbol{K} U$ $\equiv[x] Y_{1}$. Then $Y_{1} \equiv U$ and does not contain $x$. Either $R_{2} \equiv \mathbf{I}$ or $R_{2} \equiv \boldsymbol{K} B$. In the first case, $X_{2} \equiv I$, so $Y_{2} \equiv x$. But then $X \equiv[x] U x \equiv U$, which is impossible. In the second case, $X_{2} \equiv \boldsymbol{K} V$ and $Y_{2} \equiv V$ so that $Y$ does not contain $x$, and $X \equiv[x] Y \equiv \boldsymbol{K} Y$, contrary to hypothesis. Thus, $\mathrm{S} R_{1} R_{2}$ is not basic.

We may now suppose that $\mathbf{S} R_{1} R_{2} \equiv[x] P_{i j k}$ for a redex scheme $P$, so that $X \equiv[U / A][V / B][W / C][x] P_{i j k}$. Since $X \equiv[x] Y, X$ does not contain $x$, and hence, neither do $U, V, W$. Thus, Lemma 3 applies and $X \equiv[x] Q$, where $Q \equiv\left[U^{i} / A\right]\left[V^{j} / B\right]\left[W^{k} / C\right] P$. Moreover, we must have $Q \equiv Q_{1} Q_{2}$ and $X \equiv \boldsymbol{S}\left([x] Q_{1}\right)\left([x] Q_{2}\right)$, for the alternative is that $Q \equiv \mathbf{S} U^{1} V^{1} x \equiv \mathbf{S} U V x$, so that $\mathbf{S} R_{1} R_{2} \equiv \mathbf{S} A B$, which is not a redex scheme. Then by Lemma $4, Q \triangleright Y, Q_{1} \triangleright Y_{1}$, and $Q_{2} \triangleright Y_{2}$.

If $P$ is weakly irreducible, then Lemma 2 (with $Y \equiv R$ ) implies that $Y \equiv\left[U^{i^{\circ}} / A\right]\left[V^{j^{\circ}} / B\right]\left[W^{k^{\circ}} / C\right] P$. This contradicts the hypothesis that $Y$ is not a redex.

If $P$ is weakly reducible, then either $P \equiv \boldsymbol{S} A B C$ or $P \equiv \boldsymbol{K} A B$, so that either $Q \equiv \boldsymbol{S} U^{i} V^{j} W^{k}$ or $Q \equiv \boldsymbol{K} U^{i} V^{j}$. But since $Q_{1} \triangleright Y_{1}$, either $\boldsymbol{S} U^{i} V^{j} \triangleright Y_{1}$ and hence $Y_{1} \equiv \boldsymbol{S} Y^{\prime} Y^{\prime \prime}$, or else $\boldsymbol{K} U^{i} \triangleright Y_{1}$, and hence $Y_{1} \equiv \boldsymbol{K} Y^{\prime}$. In either case, $Y$ is not weakly irreducible. This final contradiction completes the proof.

\section{REFERENCES}

1. H. B. Curry and R. Feys, Combinatory logic. Vol. 1, North-Holland, Amsterdam, 1958. MR $20 \# 817$.

2. H. B. Curry, Combinatory logic. Vol. 2, with the collaboration of R. Hindley and J. P. Seldin (in preparation).

3. R. Hindley, Axioms for strong reduction in combinatory logic, J. Symbolic Logic 32 (1967), 224-236. MR 35 \#5320.

4. B. Lercher, The decidability of Hindley's axioms for strong reduction, J. Symbolic Logic 32 (1967), 237-239. MR 35 \#5321.

5. J. B. Rosser, A mathematical logic without rariables, Ann. of Math. (2) 36 (1935), $127-150$.

University College of Swansea, Wales and

State University of New York at Binghamton 